\title{
Forcing Strong Convergence of a Mann-Based Iteration for Nonexpansive and Monotone Operators in a Hilbert Space
}

\author{
Songtao Lv iD \\ School of Mathematics and Statistics, Shangqiu Normal University, Shangqiu, China \\ Correspondence should be addressed to Songtao Lv; sqlvst@yeah.net
}

Received 24 December 2021; Accepted 18 January 2022; Published 10 February 2022

Academic Editor: Sun Young Cho

Copyright (C) 2022 Songtao Lv. This is an open access article distributed under the Creative Commons Attribution License, which permits unrestricted use, distribution, and reproduction in any medium, provided the original work is properly cited.

Mann iteration is weakly convergent in infinite dimensional spaces. We, in this paper, use the nearest point projection to force the strong convergence of a Mann-based iteration for nonexpansive and monotone operators. A strong convergence theorem of common elements is obtained in an infinite dimensional Hilbert space. No compact conditions are needed.

\section{Introduction: Preliminaries}

In the real world, there are a lot of nonlinear phenomena, which can be modelled into variational inequalities and variational inclusions, such as signal processing, image recovery, and machine learning; see, e.g., [1-7] and the references therein. Fixed point methods are powerful and popular for dealing various nonlinear operator equations and inequalities in abstract spaces, in particular, for variational inequalities and variational inclusions. Recently, various efficient fixed point methods have been introduced and investigated; see, e.g., [8-13] and the references therein. Let $T$ be a nonlinear operator on a Hilbert space $H$, which is endowed with inner product $\langle\cdot, \cdot\rangle$ and induced norm $\|\cdot\|$. The fixed point set of $T$ is presented by $\operatorname{Fix}(T)$. Recall that $T$ is said to be contractive iff there is a real number $a \in(0,1)$ such that

$$
\|T x-T y\| \leq a\|x-y\|, \quad \forall x, y \in H .
$$

Recall that $T$ is said to be nonexpansive iff

$$
\|T x-T y\| \leq\|x-y\|, \quad \forall x, y \in H .
$$

Recall that $T$ is said to be firmly nonexpansive iff

$$
\|T x-T y\|^{2} \leq\langle x-y, T x-T y\rangle, \quad \forall x, y \in H .
$$

It is clear that the class of firmly nonexpansive mappings is a special class of nonexpansive mappings. One knows the projection operator (see below) is firmly nonexpansive. The class of nonexpansive operators is significant in various nonlinear equations and mathematical programming computation. It also has wide real applications in applied and industrial fields. For various iterative methods, Mann iteration is popular for dealing with fixed points of nonexpansive operators. It reads

$$
x_{n+1}=\left(1-\alpha_{n}\right) T x_{n}+\alpha_{n} x_{n},
$$

where $\left\{\alpha_{n}\right\}$ is a real number sequence in the interval $(0,1)$. However, the Mann iteration is weakly convergent only in infinite dimensional spaces; see, e.g., [14] and the references therein. To force the strong convergence without possible compact assumptions, various regularized methods have been investigated in Hilbert spaces and Banach spaces recently; see, e.g., [15-19] and the references therein. One of the efficient regularized methods is the Halpern iteration, which reads

$$
x_{n+1}=\left(1-\alpha_{n}\right) T x_{n}+\alpha_{n} x,
$$

where $\left\{\alpha_{n}\right\}$ is a real number sequence in the interval $(0,1)$ and $x$ is a fixed anchor. With some conditions on $\left\{\alpha_{n}\right\}$, it was proved that $\left\{x_{n}\right\}$ converges to $x$, which is a special fixed point 
of $T$, that is, the nearest point in $\operatorname{Fix}(T)$ to $x$. Halpern [20] pointed out that conditions (c1) $\alpha_{n} \longrightarrow 0$ as $n \longrightarrow \infty$ and (c2) $\sum_{n=1}^{\infty} \alpha_{n}=\infty$ are necessary if the Halpern iteration scheme converges in norm. In view of (c2), the Halpern iteration may not be a fast iteration. Recently, a number of researchers investigated the problem of removing (c2) with the aid of projections; see, e.g., [21-24] and the references therein. In 2000, Moudafi [25] further proposed the viscosity approximation iteration, which reads as follows:

$$
x_{n+1}=\left(1-\alpha_{n}\right) T x_{n}+\alpha_{n} S x_{n}
$$

where $S$ is a contraction. This approximation method, which improves the property of the class of nonexpansive mappings, is popular from the viewpoint of variational inequalities. Indeed, the fixed point also solves a monotone variational inequality with $S$. Another popular regularized method is the hybrid projection method, which was considered by Nakajo and Takahashi [18] for fixed points of nonexpansive mappings first. Indeed, they studied the following algorithm:

$$
\left\{\begin{array}{l}
x_{0} \in C, \\
y_{n}=\left(1-\alpha_{n}\right) T x_{n}+\alpha_{n} x_{n}, \\
Q_{n}=\left\{x \in C:\left\langle x_{n}-x, x_{n}-x_{0}\right\rangle \leq 0\right\}, \\
C_{n}=\left\{x \in C:\left\|x-y_{n}\right\| \leq\left\|x-x_{n}\right\|\right\}, \\
x_{n+1}=\operatorname{Proj}_{Q_{n} \cap C_{n}} x_{0},
\end{array}\right.
$$

where $C$ is a closed, convex, and nonempty subset of $H$ and $\operatorname{Proj}_{Q_{n} \cap C_{n}}$ is the nearest point projection onto the intersection set. They obtained a strong convergence theorem for nonexpansive mappings in a real Hilbert spaces without compact assumption on $T$. For more general nonlinear mappings though the projection-based method, we refer to [26-30] and the references therein.

Let $C$ be a convex and closed subset of a real Hilbert space $H$. From now on, $\operatorname{Proj}_{C}$ is borrowed to denote the nearest projection onto subset $C$, i.e., $\operatorname{Proj}_{C}(x):=\arg \min \{\|x-y\|, y \in C\}$. Let $A$ be a nonlinear mapping on $H$. Recall that $A$ is said to be

(1) Strongly monotone iff there exists a positive constant $\xi$ such that $\langle A x-A y, x-y\rangle \geq \xi\|x-y\|^{2}, \forall x, y \in H$

(2) Monotone iff $\langle A x-A y, x-y\rangle \geq 0, \forall x, y \in H$

(3) Cocoercive iff there exists a positive constant $\forall x, y \in H$ such that $\langle A x-A y, x-y\rangle \geq \xi \| A x-$ $A y \|^{2}, \forall x, y \in H$

Let $B: H \rightrightarrows H$ be a multivalued nonlinear mapping. Next, we turn our attention to the class of multivalued mappings. $B$ is said to be a monotone mapping if and only if for all $x, y \in H, f \in B y$, and $e \in B x \Rightarrow\langle e-f, x-x\rangle>0$. The symbol $B^{-1}(0)$ is used to stand for the set of zero points of $B$. Mapping $B$ is said to be a maximally monotone mapping iff the graph of $B, \operatorname{Graph}(B)$, is not contained in the graph of any other monotone mapping properly. Let $J_{\beta}^{B}=(I d+\beta B)^{-1}$, where $I d$ is the identity mapping and $\beta$ is a constant. This operator is called the resolvent of $B$. Its domain is denoted by $\operatorname{Dom}(B)$ in this paper. It is clear $B^{-1}(0)=\operatorname{Fix}\left(J_{\beta}^{B}\right)$.
Consider the following variational inclusion problem, which finds a point $x \in C$ such that $x \in(B+A)^{-1}(0)$, where $B$ is a multivalued maximally monotone mapping and $A$ is a $\xi$-cocoercive mapping. For the inclusion problem, splitting methods (FB, PR, and DR) are popular for zero points of the sum of the monotone mappings. Splitting methods were considered by many authors for image recovery, signal processing, and machine learning. The FB-type splitting method means an iterative method for which each iteration involves only with the individual operators not the sum. In this paper, with the condition that the solution set is nonempty, we consider finding a $\theta \in C$ such that $\theta \in F(T) \cap(B+A)^{-1}(0)$, where $T$ is a nonexpansive mapping with a nonempty fixed point set, $B$ is a multivalued maximally monotone mapping, and $A$ is a $\xi$-cocoercive mapping. We establish a strong convergence with the aid of hybrid projection and FB splitting in a Hilbert space. Our strong convergence theorem requires less restriction on parameter sequences and the operators.

To show our main findings, we also need the following necessary tools.

The nearest point projection operator $\operatorname{Proj}_{C}$ has the following property:

$\left\|\operatorname{Proj}_{C} y-\operatorname{Proj}_{C} x\right\|^{2} \leq\left\langle y-x, \operatorname{Proj}_{C} y-\operatorname{Proj}_{C}(x)\right\rangle, \quad \forall x, y \in H$.

Lemma 1 (see [31]). Let $H$ be a Hilbert space, and let $C$ be a convex, closed, and nonempty subset of $H$. Let $T$ be a nonexpansive mapping on $C$. Then, Fix $(T)$ is convex and closed.

Remark 1. Let $H$ be a Hilbert space, and let $C$ be a convex, closed, and nonempty subset of $H$. Let $A: C \longrightarrow H$ be a $\xi$-cocoercive mapping, and let $B: H \rightrightarrows H$ be a multivalued maximally monotone operator. Then, $\operatorname{Fix}\left(J_{\beta}^{B}(I d-\beta A)\right)=$ $(B+A)^{-1}(0)$, where $\beta$ is some constant and $I d$ is the identity mapping. Besides, the resolvent is firmly nonexpansive. From Lemma 1 , we have that $(B+A)^{-1}(0)$ is convex and closed.

Lemma 2 (see [31]). Let $H$ be a Hilbert space, and let $C$ be a convex, closed, and nonempty subset of $H$. Let $T$ be a nonexpansive mapping on C. Then, $I d-T$ is demiclosed (let $\left\{x_{n}\right\}$ be a sequence weakly converging to $x$, and let $T x_{n}-x_{n} \longrightarrow \infty$ be $n \longrightarrow \infty$. Then, $x$ is a fixed point of $T$ ).

\section{Main Results}

Theorem 1. Assume that $H$ is a Hilbert space and $C$ is a convex and closed subset in space $H$. Assume that $A$ is a single-valued $\xi$-cocoercive mapping from set $C$ to space $H$ and $B$ is a set-valued maximally monotone mapping from $\mathrm{H}$ to $\mathrm{H}$. Assume that $T$ is a nonexpansive mapping from $C$ to $C$, and $\operatorname{CSS}(B, A, T)=(B+A)^{-1}(0) \cap$ Fix $(T)$ is nonempty. Assume that $\left\{\alpha_{n}\right\}$ and $\left\{\beta_{n}\right\}$ are positive real number sequences. Let $\left\{x_{n}\right\}$ be a sequence in set $C$ generated in the following iterative process: 


$$
\left\{\begin{array}{l}
x_{0} \in C, \\
y_{n}=\left(1-\alpha_{n}\right) T x_{n}+\alpha_{n} J_{\beta_{n}}^{B}\left(x_{n}-\beta_{n} A x_{n}\right), \\
Q_{n}=\left\{x \in C:\left\langle x_{n}-x, x_{n}-x_{0}\right\rangle \leq 0\right\}, \\
C_{n}=\left\{x \in C:\left\|x-y_{n}\right\| \leq\left\|x-x_{n}\right\|\right\}, \\
x_{n+1}=\operatorname{Proj}_{Q_{n} \cap C_{n}} x_{0},
\end{array}\right.
$$

where $J_{\beta_{n}}^{B}$ is the resolvent mapping $\left(I d+\beta_{n} B\right)^{-1}$. Assume that $\left\{\alpha_{n}\right\}$ and $\left\{\beta_{n}\right\}$ satisfy the conditions (i) $1>\alpha_{n} \geq \alpha>0$ with $\alpha$ being a fixed real number and (ii) $0<\beta \leq \beta_{n} \leq \beta^{\prime}<2 \xi$ with $\beta$ and $\beta^{\prime}$ being two fixed real numbers. Then, the sequence $\left\{x_{n}\right\}$ converges strongly to $\operatorname{Proj}_{C S S(A, B, T)} x_{0}$.

Proof. From Lemma 1, we have that $\operatorname{Fix}(T)$ is convex and closed. From Remark 1, we have that $(B+A)^{-1}(0)$ is convex and closed. Hence, $\operatorname{CSS}(B, A, T)$ is convex and closed. This shows that the metric (nearest point) projection onto the set is well-defined.

Note that $\left\|x-y_{n}\right\|^{2} \leq\left\|x-x_{n}\right\|^{2}$ is equivalent to $2\left\langle x, x_{n}-\right.$ $\left.y_{n}\right\rangle \leq\left\|x_{n}\right\|^{2}-\left\|y_{n}\right\|^{2}$. Let $x$ and $x^{\prime}$ be the points in $C_{n}$. Then,

$$
\begin{gathered}
2 r\left\langle x, x_{n}-y_{n}\right\rangle \leq r\left(\left\|x_{n}\right\|^{2}-\left\|y_{n}\right\|^{2}\right), \\
2(1-r)\left\langle x^{\prime}, x_{n}-y_{n}\right\rangle \leq(1-r)\left(\left\|x_{n}\right\|^{2}-\left\|y_{n}\right\|^{2}\right),
\end{gathered}
$$

where $r$ is a real number in $(0,1)$. Adding the two inequalities above, we have

$$
2\left\langle r x+(1-r) x^{\prime}, x_{n}-y_{n}\right\rangle \leq\left\|x_{n}\right\|^{2}-\left\|y_{n}\right\|^{2},
$$

that is,

$$
\left\|r x+(1-r) x^{\prime}-y_{n}\right\| \leq\left\|r x+(1-r) x^{\prime}-x_{n}\right\| .
$$

It shows that $r x+(1-r) x^{\prime} \in C_{n}$. $C_{n}$ is convex. The closedness of $C_{n}$ is obvious. The definition of $\xi$-cocoercive mappings send us to the situation $\mathrm{Id}-\beta_{n} A$ is a nonexpansive mapping for each $n$. Indeed, for any $w, v \in C$,

$$
\begin{aligned}
& \left\|\left(I d-\beta_{n} A\right) w-\left(I d-\beta_{n} A\right) v\right\|^{2} \\
& =\beta_{n}^{2}\|A w-A v\|^{2}-2 \beta_{n}\langle A w-A v, w-v\rangle+\|w-v\|^{2} \\
& \leq \beta_{n}\left(\beta_{n}-2 \xi\right)\|A w-A v\|^{2}+\|w-v\|^{2} .
\end{aligned}
$$

This indicates Id $-\beta_{n} A$ is a mapping of nonexpansive. Observe that $\operatorname{CSS}(B, A, T) \subset C_{n}$. Indeed, from the nonexpansivity of the resolvent, we have

$$
\begin{aligned}
\left\|y_{n}-p\right\| & \leq\left(1-\alpha_{n}\right)\left\|T x_{n}-p\right\|+\alpha_{n}\left\|J_{\beta_{n}}^{B}\left(x_{n}-\beta_{n} A x_{n}\right)-p\right\| \\
& =\left(1-\alpha_{n}\right)\left\|T x_{n}-T p\right\|+\alpha_{n}\left\|J_{\beta_{n}}^{B}\left(x_{n}-\beta_{n} A x_{n}\right)-J_{\beta_{n}}^{B}\left(p-\beta_{n} A p\right)\right\| \\
& \leq\left(1-\alpha_{n}\right)\left\|x_{n}-p\right\|+\alpha_{n}\left\|\left(\operatorname{Id}-\beta_{n} A\right) x_{n}-\left(\operatorname{Id}-\beta_{n} A\right) p\right\| \\
& \leq\left\|x_{n}-p\right\|, \quad \forall p \in \operatorname{CSS}(A, B, T) .
\end{aligned}
$$

So, we complete the proof $\operatorname{CSS}(A, B, T) \subset C_{n}$.

On the contrary, it is obvious that $Q_{n}$ is convex and closed. Next, one shows that $\operatorname{CSS}(B, A, T) \subset Q_{n} \cap C_{n}$. Borrowing $C_{0}=C$, we have $\operatorname{CSS}(B, A, T) \subset Q_{0} \cap C_{0}$. Let $x_{m}$ be a given vector, and $\operatorname{CSS}(B, A, T) \subset Q_{m} \cap C_{m}$ for some positive integer $m$. There is a vector $x_{m+1} \in Q_{m} \cap C_{m}$ with $x_{m+1}=\operatorname{Proj}_{Q_{m} \cap C_{m}} x_{0}$. There holds $\left\langle x_{0}-x_{m+1}, x_{m+1}-j\right\rangle \geq 0$ for all $j \in Q_{m} \cap C_{m}$. Borrowing $\operatorname{CSS}(B, A, T) \subset Q_{m} \cap C_{m}$, we get $\operatorname{CSS}(B, A, T) \subset Q_{m+1}$. Thus, $\operatorname{CSS}(B, A, T) \subset Q_{m+1} \cap$ $C_{m+1}$. Hence, $\operatorname{CSS}(B, A, T) \subset Q_{n} \cap C_{n}$ for all $n$.

One next observes that $x_{n}$ is a bounded sequence. As we have showed that $\operatorname{CSS}(B, A, T)$ is convex and closed set in $C$, a unique vector $\mu \in \operatorname{CSS}(A, B, T)$ with $\mu=\operatorname{Proj}_{\operatorname{CSS}(A, B, T)} x_{0}$ is guaranteed. We have the construction of $x_{n+1}$, that is, $\operatorname{Proj}_{Q_{n} \cap C_{n}} x_{0}=x_{n+1}$. So,

$$
\left\|x_{0}-x_{n+1}\right\| \leq\left\|x_{0}-v\right\|,
$$

for each $v \in Q_{n} \cap C_{n}$. By $\mu \in \operatorname{CSS}(A, B, T) \subset Q_{n} \cap C_{n}$, we obtain

$$
\left\|x_{0}-x_{n+1}\right\| \leq\left\|x_{0}-\mu\right\|
$$

that infers $x_{n}$ is a bounded sequence. Our next step shows $\left\|x_{n+1}-x_{n}\right\| \longrightarrow 0$ as $n \longrightarrow \infty$. Because $x_{n}=\operatorname{Proj}_{Q_{n}} x_{0}$ and $x_{n+1} \in Q_{n} \cap C_{n} \subset Q_{n}$, one infers that

$$
\left\|x_{0}-x_{n}\right\| \leq\left\|x_{0}-x_{n+1}\right\| \text {. }
$$

Borrowing the conclusion ( $x_{n}$ is a bounded sequence), one infers that the limit of $\left\{\left\|x_{0}-x_{n}\right\|\right\}$ exists. We may suppose that $\lim _{n \longrightarrow \infty}\left\|x_{0}-x_{n}\right\|=d>0$. Observe

$$
\begin{aligned}
& \left\|x_{n+1}-x_{0}\right\|^{2}-\left\|x_{0}-x_{n}\right\|^{2} \\
& \geq\left\|x_{n+1}-x_{0}\right\|^{2}-\left\|x_{0}-x_{n}\right\|^{2}-2\left\langle x_{0}-x_{n}, x_{n}-x_{n+1}\right\rangle \\
& =\left\|x_{n}-x_{0}\right\|^{2}+\left\|x_{0}-x_{n+1}\right\|^{2}+2\left\langle x_{n}-x_{0}, x_{0}-x_{n+1}\right\rangle \\
& =\left\|x_{n}-x_{n+1}\right\|^{2} \geq 0,
\end{aligned}
$$

thanks to $\left\langle x_{0}-x_{n}, x_{n}-x_{n+1}\right\rangle \geq 0 \quad\left(x_{n+1} \in Q_{n}\right.$ and the property of the metric projection). By the limit of the limit of $\left\{\left\|x_{0}-x_{n}\right\|\right\}$, one infers $\lim _{n \longrightarrow \infty}\left\|x_{n+1}-x_{n}\right\|^{2}=0$.

Note that $x_{n+1}$ is in $C_{n}$. So,

$$
\left\|x_{n+1}-y_{n}\right\| \leq\left\|x_{n+1}-x_{n}\right\| \text {. }
$$


That indicates that $x_{n+1}-y_{n} \longrightarrow 0$ as $n \longrightarrow \infty$. Fur- $z_{n}=J_{\beta_{n}}^{B}\left(x_{n}-\beta_{n} A x_{n}\right)$. For any $p \in \operatorname{CSS}(B, A, T)$, $\xi$-cocoerthermore, $\quad x_{n}-y_{n} \longrightarrow 0 \quad$ as $\quad n \longrightarrow \infty$. Let cive and resolvent operators send us to

$$
\begin{aligned}
& \left\|p-z_{n}\right\|^{2} \\
& \leq\left\|J_{\beta_{n}}^{B}\left(p-\beta_{n} A p\right)-J_{\beta_{n}}^{B}\left(x_{n}-\beta_{n} A x_{n}\right)\right\|^{2} \\
& \leq\left\|\left(p-\beta_{n} A p\right)-\left(x_{n}-\beta_{n} A x_{n}\right)\right\|^{2} \\
& \leq\left\|p-x_{n}\right\|^{2}-\left(2 \xi-\beta_{n}\right) \beta_{n}\left\|A p-A x_{n}\right\|^{2} .
\end{aligned}
$$

So,

$$
\begin{aligned}
& \left\|p-y_{n}\right\|^{2} \\
& \leq\left(1-\alpha_{n}\right)\left\|T x_{n}-p\right\|^{2}+\alpha_{n}\left\|z_{n}-p\right\|^{2} \\
& \leq\left(1-\alpha_{n}\right)\left\|T x_{n}-T p\right\|^{2}+\alpha_{n}\left(\left\|p-x_{n}\right\|^{2}-\left(2 \xi-\beta_{n}\right) \beta_{n}\left\|A p-A x_{n}\right\|^{2}\right) \\
& \leq\left\|p-x_{n}\right\|^{2}-\alpha_{n}\left(2 \xi-\beta_{n}\right) \beta_{n}\left\|A p-A x_{n}\right\|^{2} .
\end{aligned}
$$

That is,

$\alpha_{n}\left(2 \xi-\beta_{n}\right) \beta_{n}\left\|A p-A x_{n}\right\|^{2} \leq\left\|y_{n}-x_{n}\right\|\left(\left\|p-x_{n}\right\|+\left\|p-y_{n}\right\|\right)$.
By the fact that $\left\|y_{n}-x_{n}\right\| \longrightarrow 0$ as $n \longrightarrow \infty$, we have $A x_{n}-A p \longrightarrow 0$ as $n \longrightarrow \infty$. By the firm nonexpansivitity of the resolvent operator, we also have

$$
\begin{aligned}
& \left\|p-z_{n}^{2}\right\| \\
& \leq\left\langle p-z_{n},\left(p-\beta_{n} A p\right)-\left(x_{n}-\beta_{n} A x_{n}\right)\right\rangle \\
& =\frac{1}{2}\left(\left\|p-x_{n}\right\|^{2}+\left\|p-z_{n}\right\|^{2}-\left\|x_{n}-z_{n}-\beta_{n}\left(A p-A x_{n}\right)\right\|^{2}\right) \\
& \leq \frac{1}{2}\left(\left\|p-x_{n}\right\|^{2}+\left\|p-z_{n}\right\|^{2}-\left\|x_{n}-z_{n}\right\|^{2}-\beta_{n}^{2}\left\|A p-A x_{n}\right\|^{2}+2 \beta_{n}\left\|x_{n}-z_{n}\right\|\left\|A p-A x_{n}\right\|\right) \\
& \leq \frac{1}{2}\left(\left\|p-x_{n}\right\|^{2}+\left\|p-z_{n}\right\|^{2}-\left\|x_{n}-z_{n}\right\|^{2}+2 \beta_{n}\left\|x_{n}-z_{n}\right\|\left\|A p-A x_{n}\right\|\right),
\end{aligned}
$$

which holds that

$$
\begin{aligned}
\left\|p-z_{n}\right\|^{2} & \leq\left\|p-x_{n}\right\|^{2}-\left\|x_{n}-z_{n}\right\|^{2}+2 \beta_{n}\left\|x_{n}-z_{n}\right\|\left\|A p-A x_{n}\right\|, \\
\left\|p-y_{n}\right\|^{2} & \leq\left(1-\alpha_{n}\right)\left\|T x_{n}-T p\right\|^{2}+\alpha_{n}\left\|J_{\beta_{n}}^{B}\left(x_{n}-\beta_{n} A x_{n}\right)-p\right\|^{2} \\
& \leq\left(1-\alpha_{n}\right)\left\|x_{n}-p\right\|^{2}+\alpha_{n}\left\|z_{n}-p\right\|^{2} \\
& \leq\left\|x_{n}-p\right\|^{2}-\alpha_{n}\left\|x_{n}-z_{n}\right\|^{2}+2 \beta_{n} \alpha_{n}\left\|x_{n}-z_{n}\right\|\left\|A p-A x_{n}\right\| .
\end{aligned}
$$


So, $\quad \alpha_{n}\left\|x_{n}-z_{n}\right\|^{2} \leq\left\|x_{n}-y_{n}\right\| C+2 \beta_{n} \alpha_{n}\left\|x_{n}-z_{n}\right\| \| A p-$ $A x_{n} \|$, where $C$ is some constant. By the requirement on the control parameter and the result that $A p-A x_{n} \longrightarrow \infty$ as $n \longrightarrow \infty$ and $x_{n}-z_{n} \longrightarrow \infty$ as $n \longrightarrow \infty$. With a simple calculation, we have $x_{n}-T x_{n} \longrightarrow \infty$ as $n \longrightarrow \infty$. We have the fact that $\left(\operatorname{Id}-\beta_{n} A\right) x_{n} \in\left(\operatorname{Id}+\beta_{n} B\right) z_{n}$. It holds

$$
\frac{x_{n}-z_{n}}{\beta_{n}}-A x_{n} \in B z_{n} \text {. }
$$

By the assumption that $B$ is maximally monotone,

$$
\left\langle\frac{x_{n}-z_{n}}{\beta_{n}}-A x_{n}-u, z_{n}-v\right\rangle \geq 0,
$$

for any $u \in B v$. By the result that $\left\{x_{n}\right\}$ is a bounded sequence, there is a subsequence $\left\{x_{n_{m}}\right\}$ converges to $\theta$ weakly. The $\xi$-cocoercive mappings yield $A x_{n_{m}} \longrightarrow A \theta$. It holds $\langle-A \theta-$ $u, \theta-v\rangle \geq 0$. It shows $0 \in(B+A)(\theta)$. Note that Id $-T$ is demiclosed (Lemma 2). One asserts $\theta \in \operatorname{Fix}(T)$. One next shows that $\theta=\operatorname{Proj}_{\operatorname{CSS}(B, A, T)} x_{0}$ and $x_{n}$ converges to it strongly. Set $\bar{x}=\operatorname{Proj}_{\operatorname{CSS}(B, A, T)} x_{0}$. Since the functional $\|\cdot\|$ is weakly lower semicontinuous, one has

$$
\left\|\bar{x}-x_{0}\right\| \leq\left\|\theta-x_{0}\right\| \leq \liminf _{m \longrightarrow \infty}\left\|x_{0}-x_{n_{m}}\right\| \leq \limsup _{m \longrightarrow \infty}\left\|x_{0}-x_{n_{m}}\right\| \leq\left\|\bar{x}-x_{0}\right\| .
$$

One gets $\left\|\bar{x}-x_{0}\right\|=\lim _{m \longrightarrow \infty}\left\|x_{0}-x_{n_{m}}\right\|=\left\|\theta-x_{0}\right\|$. Let Since the framework is a Hilbert space, one gets $x_{n} \longrightarrow \theta$ as $n \longrightarrow \infty$. This finishes this theorem.

$$
\partial f(x)=\{z \in H: f(x)+\langle y-x, z\rangle \leq f(y), \forall y \in H\}, \quad \forall, x \in H,
$$

where $f: H \longrightarrow(-\infty, \infty]$ is a proper, convex, and lower semicontinuous function. Rockfellar [32] proved that $\partial f$ is a multivalued maximally monotone operator. Let $C$ be a closed, convex, and nonempty subset of $H$ and $i_{C}$ be the indicator function of $C$, that is,

$$
i_{C} x= \begin{cases}0, & x \in C, \\ \infty, & x \notin C .\end{cases}
$$

Furthermore, we define the normal cone $N_{C}(v)$ of $C$ at $v$ as follows:

$$
N_{C} v=\{z \in H:\langle z, y-v\rangle \leq 0, \forall y \in H\},
$$

for any $v \in C$. Then, $i_{C}: H \longrightarrow(-\infty, \infty]$ is proper, convex, and lower semicontinuous on $H . \partial i_{C}$ is a maximally monotone operator. Let $\operatorname{Res}_{\lambda} x=\left(\mathrm{Id}+\lambda \partial i_{C}\right)^{-1} x$. So, $\partial i_{C} x=N_{C} x$ and $x \in C$; we obtain

$$
v=J_{\lambda}^{\partial i_{C}} x \Longleftrightarrow v=\operatorname{Proj}_{C} x,
$$

where $\operatorname{Proj}_{C}^{\partial i_{C}}$ is the metric projection onto $C$. This yields $x \in\left(A+\partial i_{C}\right)^{-1}(0) \Longleftrightarrow x \in \operatorname{VI}(A, C)$, where $\operatorname{VI}(A, C)$ denotes the classical variational inequality, that is, find a point $x \in C$ such that $\langle A x, y-x\rangle \geq 0$ for all $y \in C$.

Corollary 1. Assume that $H$ is a Hilbert space and $C$ is a convex and closed subset in space $H$. Assume that $A$ is a single-valued $\xi$-cocoercive mapping from set $C$ to space $H$. Assume that $T$ is a nonexpansive mapping from $C$ to $C$ and $\operatorname{VI}(A, C) \cap \operatorname{Fix}(T)$ is nonempty. Assume that $\left\{\alpha_{n}\right\}$ and $\left\{\beta_{n}\right\}$ are positive real number sequences. Let $\left\{x_{n}\right\}$ be a sequence in set $C$ generated in the following iterative process:

$$
\left\{\begin{array}{l}
x_{0} \in C, \\
y_{n}=\left(1-\alpha_{n}\right) T x_{n}+\alpha_{n} \operatorname{Proj}_{C}\left(x_{n}-\beta_{n} A x_{n}\right), \\
Q_{n}=\left\{x \in C:\left\langle x_{n}-x, x_{n}-x_{0}\right\rangle \leq 0\right\} \\
C_{n}=\left\{x \in C:\left\|x-y_{n}\right\| \leq\left\|x-x_{n}\right\|\right\} \\
x_{n+1}=\operatorname{Proj}_{Q_{n} \cap C_{n}} x_{0} .
\end{array}\right.
$$

Assume that $\left\{\alpha_{n}\right\}$ and $\left\{\beta_{n}\right\}$ satisfy the conditions (i) $1>\alpha_{n} \geq \alpha>0$ with $\alpha$ being a fixed real number and (ii) $0<\beta \leq \beta_{n} \leq \beta^{\prime}<2 \xi$ with $\beta$ and $\beta^{\prime}$ being two fixed real numbers. Then, the sequence $\left\{x_{n}\right\}$ converges strongly to $\operatorname{Proj}_{\mathrm{VI}(A, C) \cap \mathrm{Fix}(T)} x_{0}$.

\section{Data Availability}

The data used to support the findings of this study are included within the article.

\section{Conflicts of Interest}

The author declares that he has no conflicts of interest.

\section{References}

[1] N. T. An, "Robust feature selection via nonconvex sparsitybased methods," Journal of Nonlinear and Variational Analysis, vol. 5, pp. 59-77, 2021.

[2] J. Fan, X. Qin, and B. Tan, "Convergence of an inertial shadow Douglas-Rachford splitting algorithm for monotone inclusions," Numerical Functional Analysis and Optimization, pp. 1-18, 2021. 
[3] L. V. Nguyen, "Weak sharpness and finite convergence for solutions of nonsmooth variational inequalities in Hilbert spaces," Applied Mathematics and Optimization, vol. 84, no. 201, pp. 807-828, 2020.

[4] X. He, "An inertial projection neural network for solving variational inequalities," IEEE Transactions on Cybernetics, vol. 47, pp. 809-814, 2016.

[5] L. V. Nguyen and X. Qin, "The minimal time function associated with a collection of sets," ESAIM: Control, Optimisation and Calculus of Variations, vol. 26, p. 93, 2020.

[6] X. Qin and N. T. An, "Smoothing algorithms for computing the projection onto a Minkowski sum of convex sets," Computational Optimization and Applications, vol. 74, no. 3, pp. 821-850, 2019.

[7] X. Zhao and J. C. Yao, "Linear convergence of a nonmonotone projected gradient method for multiobjective optimization," Journal of Global Optimization, 2021.

[8] S. Y. Cho, "A convergence theorem for generalized mixed equilibrium problems and multivalued asymptotically nonexpansive mappings," Journal of Nonlinear and Convex Analysis, vol. 21, pp. 1017-1026, 2020.

[9] L. Liu, B. Tan, and S. Y. Cho, "On the resolution of variational inequality problems with a double-hierarchical structure," Journal of Nonlinear and Convex Analysis, vol. 21, pp. 377-386, 2020.

[10] B. A. B. Dehaish, "A regularization projection algorithm for various problems with nonlinear mappings in Hilbert spaces," Journal of Inequalities and Applications, vol. 2015, p. 51, 2015.

[11] L. Liu, S. Y. Cho, and J. C. Yao, "Convergence analysis of an inertial Tseng's extragradient algorithm for solving pseudomonotone variational inequalities and applications," Journal of Nonlinear and Variational Analysis, vol. 5, pp. 627-644, 2021.

[12] J. Fan, L. Liu, and X. Qin, “A subgradient extragradient algorithm with inertial effects for solving strongly pseudomonotone variational inequalities," Optimization, vol. 69, no. 9, pp. 2199-2215, 2020.

[13] Y. Shehu and J. N. Ezeora, "Weak and linear convergence of a generalized proximal point algorithm with alternating inertial steps for a monotone inclusion problem," Journal of Nonlinear and Variational Analysis, vol. 5, pp. 881-892, 2021.

[14] A. Genel and J. Lindenstrauss, "An example concerning fixed points," Israel Journal of Mathematics, vol. 22, no. 1, pp. 81-86, 1975.

[15] K. Aoyama, F. Kohsaka, and W. Takahashi, "Shrinking projection methods for firmly nonexpansive mappings," Nonlinear Analysis: Theory, Methods \& Applications, vol. 71, no. 12, pp. e1626-e1632, 2009.

[16] H. He and R. Chen, "Strong convergence theorems of the CQ method for nonexpansive semigroups," Fixed Point Theory and Applications, vol. 2007, Article ID 059735, 2007.

[17] Y. Kimura, "A shrinking projection method for nonexpansive mappings with nonsummable errors in a Hadamard space," Annals of Operations Research, vol. 243, no. 1-2, pp. 89-94, 2016.

[18] K. Nakajo and W. Takahashi, "Strong convergence theorems for nonexpansive mappings and nonexpansive semigroups," Journal of Mathematical Analysis and Applications, vol. 279, no. 2, pp. 372-379, 2003.

[19] Y. Kimura and W. Takahashi, "On a hybrid method for a family of relatively nonexpansive mappings in a Banach space," Journal of Mathematical Analysis and Applications, vol. 357, no. 2, pp. 356-363, 2009.
[20] B. Halpern, "Fixed points of nonexpanding maps," Bulletin of the American Mathematical Society, vol. 73, no. 6, pp. 957-961, 1967.

[21] C. Martinez-Yanes and H.-K. Xu, "Strong convergence of the CQ method for fixed point iteration processes," Nonlinear Analysis: Theory, Methods \& Applications, vol. 64, no. 11, pp. 2400-2411, 2006.

[22] U. Kohlenbach, "Quantitative analysis of a Halpern-type proximal point algorithm for accretive operators in Banach spaces," Journal of Nonlinear and Convex Analysis, vol. 21, pp. 2125-2138, 2020.

[23] Z. Wang, Y. Su, D. Wang, and Y. Dong, "A modified Halperntype iteration algorithm for a family of hemi-relatively nonexpansive mappings and systems of equilibrium problems in Banach spaces," Journal of Computational and Applied Mathematics, vol. 235, no. 8, pp. 2364-2371, 2011.

[24] J. Xiao, L. Huang, and Y. Wang, "Strong convergence of modified inertial Halpern simultaneous algorithms for a finite family of demicontractive mappings," Applied Set-Valued Analysis and Optimization, vol. 2, pp. 317-327, 2020.

[25] A. Moudafi, "Viscosity approximation methods for fixedpoints problems," Journal of Mathematical Analysis and Applications, vol. 241, no. 1, pp. 46-55, 2000.

[26] L. V. Nguyen and X. Qin, "Some results on strongly pseudomonotone quasi-variational inequalities," Set-Valued and Variational Analysis, vol. 28, pp. 239-257, 2020.

[27] S. Cho, "A monotone Bregan projection algorithm for fixed point and equilibrium problems in a reflexive Banach space," Filomat, vol. 34, no. 5, pp. 1487-1497, 2020.

[28] B. Tan, S. Li, and S. Y. Cho, "Inertial projection and contraction methods for pseudomonotone variational inequalities with non-Lipschitz operators and applications," Applicable Analysis, pp. 1-23, 2021.

[29] S.-s. Chang, J. C. Yao, C.-F. Wen, and L. J. Qin, "Shrinking projection method for solving inclusion problem and fixed point problem in reflexive Banach spaces," Optimization, vol. 70, no. 9, pp. 1921-1936, 2021.

[30] M. A. Olona, "Inertial shrinking projection algorithm with self-adaptive step size for split generalized equilibrium and fixed point problems for a countable family of nonexpansive multivalued mappings," Demonstratio Mathematica, vol. 54, pp. 47-67, 2021.

[31] W. Takahashi, Nonlinear Functional Analysis, Yokohama Publishers, Yokohama, Japan, 2000.

[32] R. T. Rockafellar, "Monotone operators and the proximal point algorithm," SIAM Journal on Control and Optimization, vol. 14, no. 5, pp. 877-898, 1976. 